\title{
JPEG2000 Part 2 Wavelet Packet Subband Structures in Fingerprint Recognition
}

\author{
Bernhard Mühlbacher, Thomas Stütz, and Andreas Uhl \\ Department of Computer Sciences, University of Salzburg, Austria
}

\begin{abstract}
The impact of using different wavelet packet subband structures in JPEG2000 on the matching accuracy of a fingerprint recognition system is investigated. In particular, we relate rate-distortion performance as measured in PSNR to the matching scores as obtained by the recognition system. Employing wavelet packets instead of the dyadic wavelet transform turns out to be of advantage only in case of low bitrates (i.e. high compression rates). For such settings, the good performance of the WSQ structure is confirmed also in JPEG2000 and in particular we get better recognition accuracy using the WSQ structure as compared to the employment of a rate-distortion optimising subband selection approach.
\end{abstract}

Keywords: fingerprint recognition, wavelet packets, JPEG2000, WSQ, rate-distortion optimisation

\section{INTRODUCTION}

With the increasing usage of biometric systems the question arises naturally how to store and handle the acquired sensor data. In this context, the compression of these data may become imperative under certain circumstances due to the large amounts of data involved. Among other possibilities (e.g. like template storage on IC cards), compression technology may be used for the transmission of sample data between sensor and matching device in distributed biometric systems, where the data acquisition stage is often dislocated from the feature extraction and matching stage (this is true for the enrolment phase as well as for authentication). In such environments with often low power and mobile acquisition devices, the sensor data have to be transferred via a network link to the respective location, often over wireless channels with low bandwidth and high latency. Therefore, a minimisation of the amount of data to be transfered is highly desirable, which is achieved by compressing the data in lossy manner before transmission.

However, the distortions introduced by compression artifacts may interfere with subsequent feature extraction and may degrade the matching results. In particular, FRR or FNMR will increase (since features of the data of legitimate users are extracted less accurately from compressed data) which in turn affects user convenience and general acceptance of the biometric system. In extreme cases, even FAR or FMR might be affected.

Optimisation of compression algorithms to meet the specific properties of the data to be compressed is a natural strategy. For example, contrasting to the optimisation of the JPEG quantisation matrix with respect to human perception as done for the development of the default matrix in the standard, rate/distortion criteria have also been used successfully for the design of this matrix. ${ }^{1}$ Compression algorithms tuned for application in the pattern recognition context have been proposed, ${ }^{2}$ which are based on the modification of the standard JPEG compression algorithm: this is done by emphasising middle and high frequencies and discarding low frequencies (the standard JPEG quantisation matrix is rotated by 180 degrees). JPEG quantisation matrix optimisation has already been considered in biometrics: a rate/distortion criterion is employed in the context of face recognition ${ }^{3}$ and superior recognition performance is achieved as compared to the standard matrix. We have designed optimised JPEG matrices for iris data compression in recent work ${ }^{4-6}$ leading to recognition performance improvements as well.

In this work, we focus on fingerprint image data. Inspired by WSQ ("wavelet scalar quantisation" as designed by the FBI for its fingerprint databases ${ }^{7}$ ), which uses a specific wavelet packet (WP) subband structure emphasising the important high frequency bands, we will apply wavelet packet subband structures ${ }^{8}$ in $\mathrm{JPEG}^{2000^{9}}$ as defined in Part 2 of the standard.

E-mail: uhl@cosy.sbg.ac.at 
The use of adapted WP basis for image compression purposes has been subject to investigation since the introduction of the first adaptation technique called "best basis algorithm" ${ }^{10}$ In this approach, a rate-independent but suboptimal selection scheme is used based on various additive information cost functions. An extension to this approach employing non-additive cost functions has been developed soon after. ${ }^{11}$ We have used genetic algorithms $^{12}$ to assess the degree of optimality and to further optimise the subband structures found by these algorithms in earlier work. The employment of rate-distortion optimisation criteria for WP subband structure selection has been first demonstrated for classical wavelet image coding schemes, ${ }^{13}$ but has been extended later even to WP zero-tree based compression algorithms. ${ }^{14}$ The first approach and some suboptimal variants thereof have been integrated into embedded block-based coding schemes recently. ${ }^{15,16}$

The employment of WP subband structures in the context of JPEG2000 Part 2 has not been subject to extensive investigations so far. In earlier work, we have assessed the variants of representing WP bases as discussed during the development of the JPEG2000 Part 2 standard with respect to compression performance ${ }^{17}$ and have proposed to use secret wavelet packet bases as a means for compression integrated JPEG2000 encryption ${ }^{18}$ (where the impact on compression performance needs to be controled). Besides using the classical Dyadic and WSQ WP structures, we integrate the classical rate distortion opimizing WP subband selection scheme ${ }^{13}$ into the JPEG2000 EBCOT coding framework applying the rate-distortion optimal basis selection to the compression of fingerprint images. Specifically, we will employ rate-distortion optimised subband structures adapted for each image.

In Section 2, we review and discuss the available literature on fingerprint image compression. Section 3 is the main part of this work where we first describe the employed image compression techniques, the used biometric recognition system, and the data these algorithms are applied to. Subsequently we present and discuss our experimental results where we compare and rank the compression algorithms in use. Section 4 concludes the paper.

\section{COMPRESSION OF FINGERPRINT DATA}

During the last decade, several algorithms and standards for compressing image data relevant in biometric systems have evolved. The certainly most relevant one is the ISO/IEC 19794 standard on Biometric Data Interchange Formats, where Parts 4, 5, and 6 cover fingerprint, face, and iris image data, respectively. ISO/IEC 19794-4 allows fingerprint image data to be stored in lossy manner in the JPEG, ${ }^{19}$ WSQ $^{7}$ and JPEG2000 ${ }^{9}$ formats, where the latter is recommended. A corresponding specific JPEG2000 Part I profile for 1000 ppi fingerprint images has been proposed. ${ }^{20}$

While the data formats specified by the ISO/IEC 19794 standard are fixed at present state, their impact on recognition accuracy as compared to other algorithms is not well investigated and documented. Also, the corresponding compression schemes are mostly employed with their respective default settings and are hardly optimzed with respect to the target image material. Moreover, many other general-purpose algorithms as well as techniques tailored to specific biometric modalities exist, which we will discuss in the following.

It is worth noticing that the evaluation of compression performance in biometric systems is surprisingly limited to PSNR or RMS-error computation and to psycho-visual studies in many investigations. Effects on actual matching rates or recognition accuracy are rarely documented, especially in the earlier papers on the topic.

Fingerprint images exhibit characteristic high energy in certain high frequency bands resulting from the ridge-valley pattern and other structures. To account for this property, the WSQ standard for lossy fingerprint compression as adopted by the $\mathrm{FBI}^{7}$ uses a specific wavelet packet subband structure which emphasises the important high frequency bands. This standard has been shown to be clearly superior to JPEG compression in terms of psycho-visual and PSNR quality. ${ }^{21}$

Wavelet-based compression schemes have been subsequently investigated in great number for their usefulness in fingerprint compression. Filter choice in classical pyramidal coding schemes specifically tuned for fingerprint compression has been one of the main areas of research. Biorthogonal filters have been identified as being superior to orthogonal ones. ${ }^{22,23}$ More specialised filters are optimised for fingerprint compression, ${ }^{24}$ e.g. a special type of wavelet filter (polyphase) to adapt to high frequency content is suggested. ${ }^{25}$ Simulated annealing over the 
wavelet filter coefficients ${ }^{26}$ is used as well as a method based on the coevolutionary genetic algorithm ${ }^{27}$ to evolve specialised wavelets for fingerprint images (the latter work with focus on the JPEG2000 context).

Inspired by the WSQ FBI algorithm, a few WP based fingerprint compression schemes have been developed. An improved WSQ entropy coding stage using lossless zerotree coding is proposed. ${ }^{28}$ Various subband structures with subsequent lattice vector quantisation as proposed ${ }^{29}$ turn out to be superior to JPEG, WSQ, EZW, and SPIHT among others. The concept of adaptive subband structures is also used to improve on the fixed structure WSQ method. ${ }^{30}$ The WP filters and the decomposition level are proposed to be adapted in addition to the selection of subbands which results in a better match of the actual frequency characteristics of the image. ${ }^{31}$

A final class of compression schemes exploits the strong directional features in fingerprint images caused by ridges and valleys. A scanning procedure following dominant ridge direction has shown to improve lossless coding results as compared to JPEG-LS and PNG. ${ }^{32}$ A wavelet footprint representation characterising efficiently singular structures (corresponding to ridges) is used ${ }^{33}$ which turns out to deliver better results as compared to the SPIHT algorithm. ${ }^{34}$ Contourlets ${ }^{35,36}$ and contourlet packets ${ }^{37}$ are used to exploit directional information which also results in improvements as compared to classical algorithms.

One of the few exceptions in designing compression schemes focusing already on optimising matching performance is found in early work ${ }^{38}$ where image tiles containing fingerprint minutiae are compressed using PCA and wavelet transform and the effects on FAR of a specific recognition system are studied. In more recent work, ${ }^{39}$ we have investigated the impact of JPEG, JPEG2000, SPIHT, PRVQ, and Fractal image compression on recognition accuracy of selected fingerprint and face recognition systems. A similar contribution ${ }^{40}$ also relates JPEG, JPEG2000, and (WSQ) compression rates to recognition performance of some fingerprint and face recognition systems.

\section{EXPERIMENTAL STUDY}

\subsection{Setting and Methods}

\subsubsection{Compression Algorithms}

For the wavelet-based JPEG2000 ${ }^{9}$ standard we have used the JAVA reference implementation JJ2000*. This software has been extended to enable the use of arbitrary WP bases in a standard conformant manner, the corresponding software is available at www.wavelab.at/sources/. We use three different compression schemes, two of which are based on a fixed subband structure: the first is the classical dyadic wavelet decomposition structure as used in JPEG2000 Part 1, the second is the subband structure as used in WSQ (the latter being implemented into the above mentioned software corresponding to JPEG2000 Part 2). Fig. 1 shows both decomposition schemes.

The third compression scheme integrates a rate-distortion optimal WP subband structure selection algorithm ${ }^{13}$ into the EBCOT-based JPEG2000 algorithm, similar to an approach described recently. ${ }^{15}$ The algorithm (denoted as RDOH) uses the rate-distortion statistics of JPEG2000 where the EBCOT scheme uses it to find the optimal truncation points for the code-blocks. In addition to the use of the classical rate information, we also consider the JPEG2000 header data during the computation of the optimal subband structure (RDOH stands for "rate-distortion optimisation with headers"). In this technique, for each fingerprint image and each rate considered, a distinct subband structure is computed, which additionally is encoded into the bitstream.

In the subsequent biometric matching, we investigate two cases:

- compressed vs. compressed case (CCC), where we match a compressed "sample image" with all compressed images "in the database" (which are compressed with the same compression-rate as the sample image, given in $[b p p])$.

- compressed vs. uncompressed case (CUC), where we match the compressed sample image with all the uncompressed images in the database.

\footnotetext{
${ }^{*}$ http://jj2000.epfl.ch/
} 


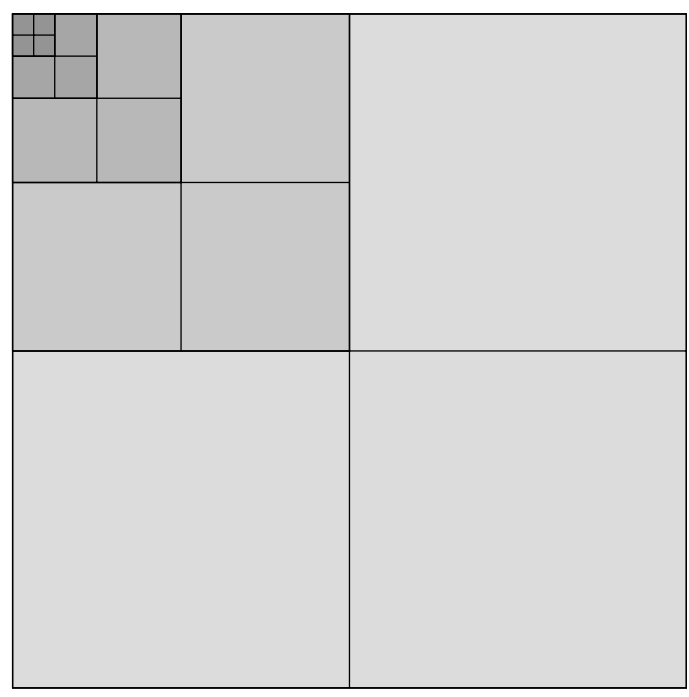

(a) Dyadic

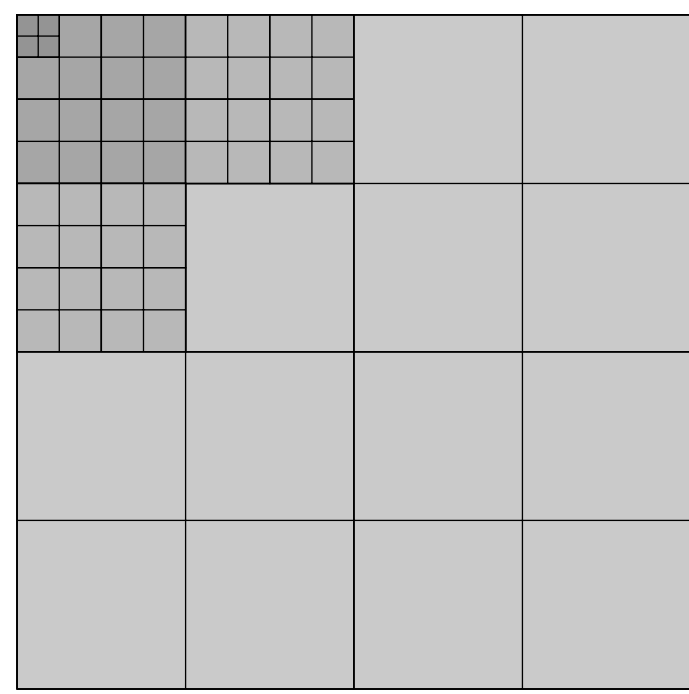

(b) WSQ

Figure 1. Fixed Wavelet Packet decomposition structures

\subsubsection{Fingerprint Recognition System}

We employ the NIST Fingerprint Image Software 2 (NFIS2) ${ }^{41}$ and apply it in a verification setting. The "MINDTCT"-tool is applied to extract the feature data from the two images subject to matching (after they have been converted from JPEG2000 format to lossless JPEG which is the required input format for MINDTCT). MINDTCT extracts minutiae feature information, i.e. ridge bifurcations and ridge terminations in this case. Subsequently, we employ the "BOZORTH3" matcher which computes the matching value between the two fingerprint images under consideration. Sweeping the matching decision threshold across a wide range of values enables us to compute the false match rate (FMR) and the false non-match rate (FNMR) for a specific compression rate, which are then combined into receiver operating characteristic (ROC) plots. Those are used to compare the different compression settings in the subsequent subsection.

\subsubsection{Fingerprint Sample Data}

As sample data, we use the 4 fingerprint databases as provided for the Fingerprint Verification Competition 2004 (FVC2004). The different sensors used to acquire the data and the corresponding resolutions are given in Table 1.

Table 1. Databases and their sensors

\begin{tabular}{|c|c|c|c|}
\hline & Sensor & Size & Resolution \\
\hline DB1 & optical sensor & $640 \times 480$ & $500 \mathrm{dpi}$ \\
\hline DB2 & optical sensor & $328 \times 364$ & $500 \mathrm{dpi}$ \\
\hline DB3 & thermal sweeping sensor & $300 \times 480$ & $512 \mathrm{dpi}$ \\
\hline DB4 & synthetic fingerprint generation & $288 \times 384$ & $500 \mathrm{dpi}$ \\
\hline
\end{tabular}

In each database, we have images of 120 fingers (forefinger and middle finger of both hands) of 30 different persons with 8 impressions each. In Figure 2, sample images for each DB are shown.

\subsection{Experimental Results}

First, we present the rate-distortion performance for the three compression schemes under investigation. In Figures 3 and 4 we plot the PSNR against the range of observed bitrates from $0.1-0.35[b p p]$ (which corresponds to compression rates $80-25)$. 

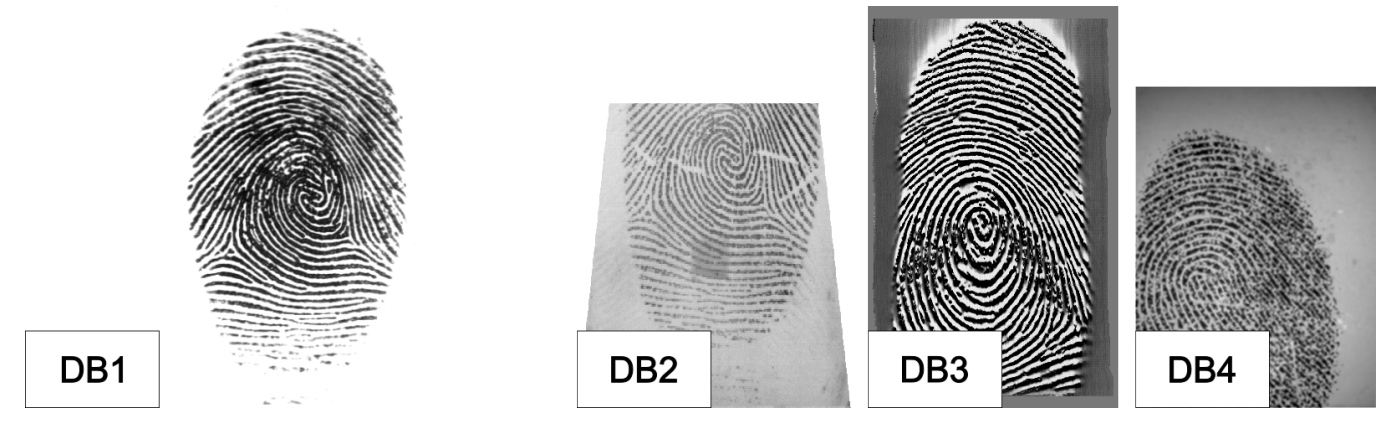

Figure 2. sample image from each database

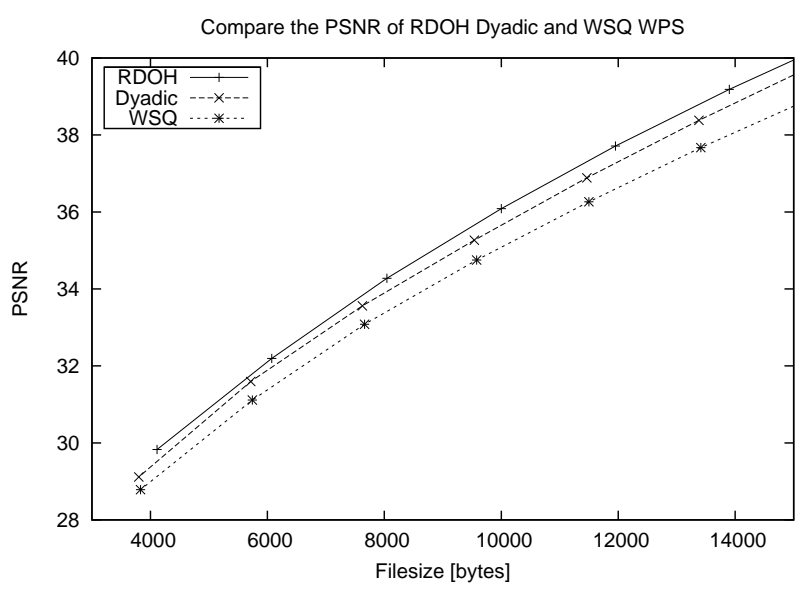

(a) DB1

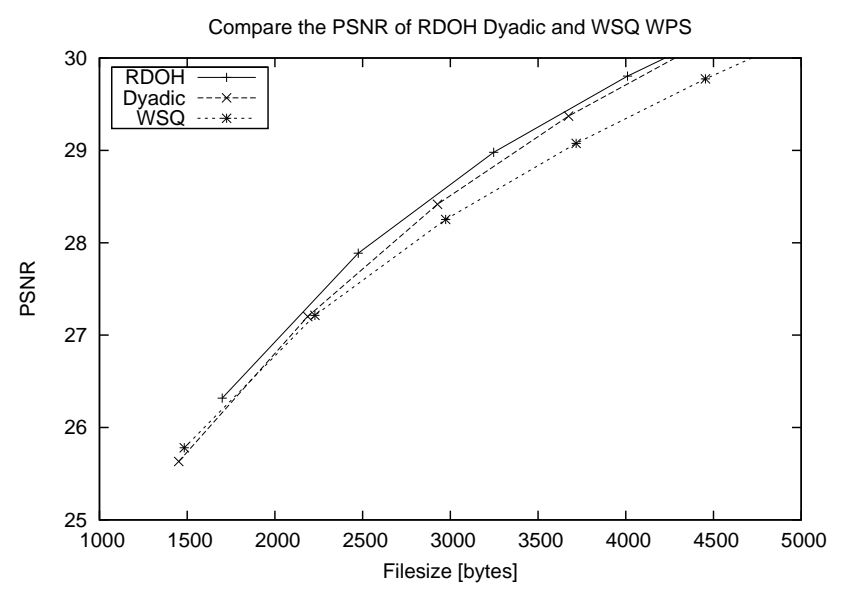

(b) DB2

Figure 3. PSNR of RDOH, WSQ and Dyadic Wavelet Packet subband structures.

As it is expected due to the rate-distortion optimal subband structure choice, RDOH is the best approach for all bitrates and databases considered (except for DB3 @ 2000 bytes). For the lowest bitrates, WSQ gets better

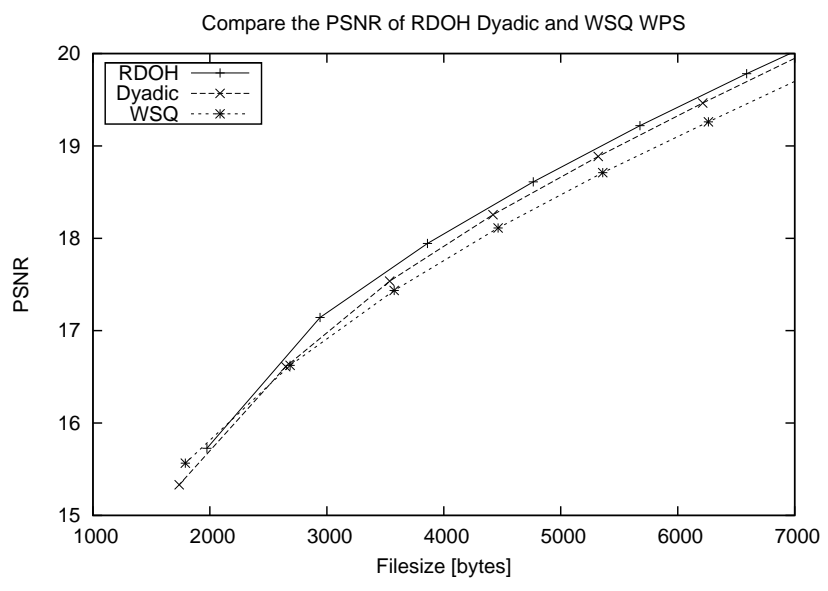

(a) DB3

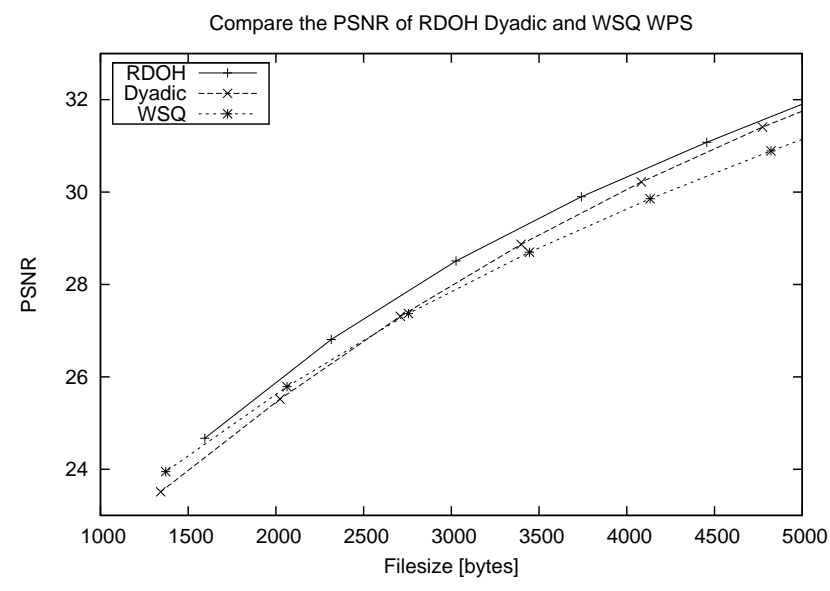

(b) DB4

Figure 4. PSNR of RDOH, WSQ and Dyadic Wavelet Packet subband structures. 
then the Dyadic subband structure, for higher bitrates the WSQ subband structure exhibits the worst PSNR values.

Now we turn to recognition accuracy as visualised in ROC curves in the following. First, we consider the CCC scenario (where both images involved in matching are compressed to the same bitrate). Fig. 5 shows averaged ROC values across all 4 databases considered, for bitrates $0.1,0.15$, and 2.5 [bpp], respectively. For increasing bitrate (and decreasing compression rate), the curves of the different compression schemes get closer together and the results are less distinctive, however, the ranking among the three compression schemes is preserved. Therefore, concerning database specific results, we only present bitrate 0.1 [bpp] in the following. In the plot, we observe WSQ taking a clear lead and the Dyadic structure being worst. This is surprising since the rate-distortion performance had predicted the opposite behaviour.

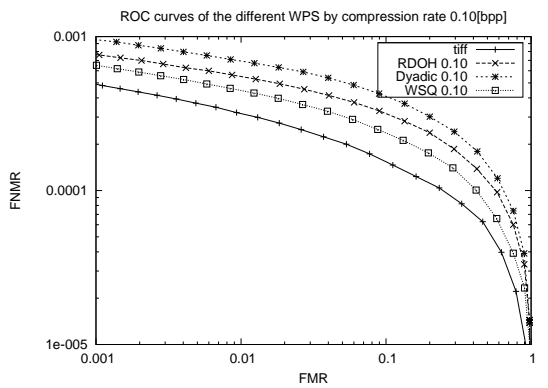

(a) $0.1 \mathrm{bpp}$

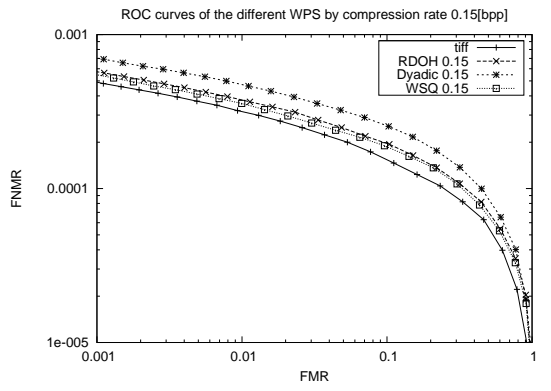

(b) $0.15 \mathrm{bpp}$

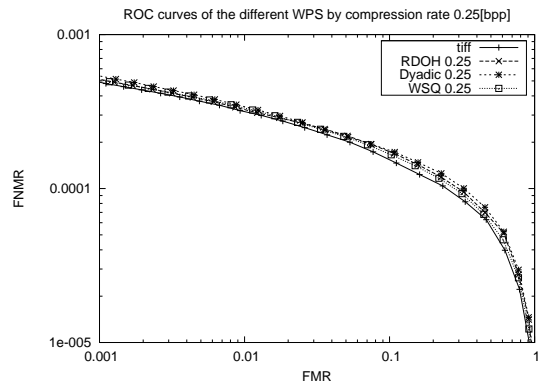

(c) $0.25 \mathrm{bpp}$

Figure 5. CCC scenario: averaged ROC of the RDOH, Dyadic and WSQ WP subband structures.

When considering the results for DB1 and DB2 in the CCC scenario (as shown in Fig. 6) we find the results for DB1 to be very close together (with the Dyadic structure being worst), WSQ is even slightly superior as compared to matching uncompressed images (which can be considered a surprise at this low bitrate). The situation is much clearer for DB2: here, WSQ is performing best across the entire range of considered FMRs, while the Dyadic structure performs always worst. When comparing this result to the rate-distortion performance (see Fig. 3), we notice that PSNR does not correspond well to the observed recognition performance since WSQ has been identified to deliver worst PSNR values but best recognition accuracy.

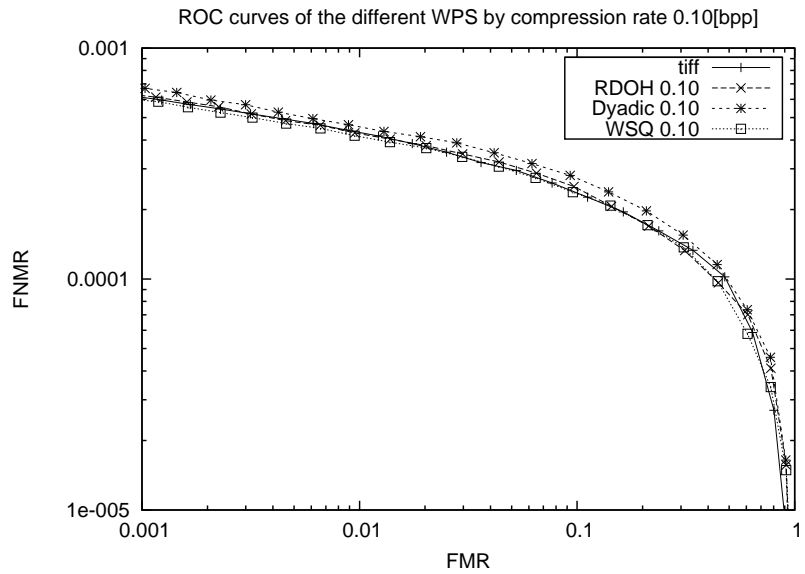

(a) DB1

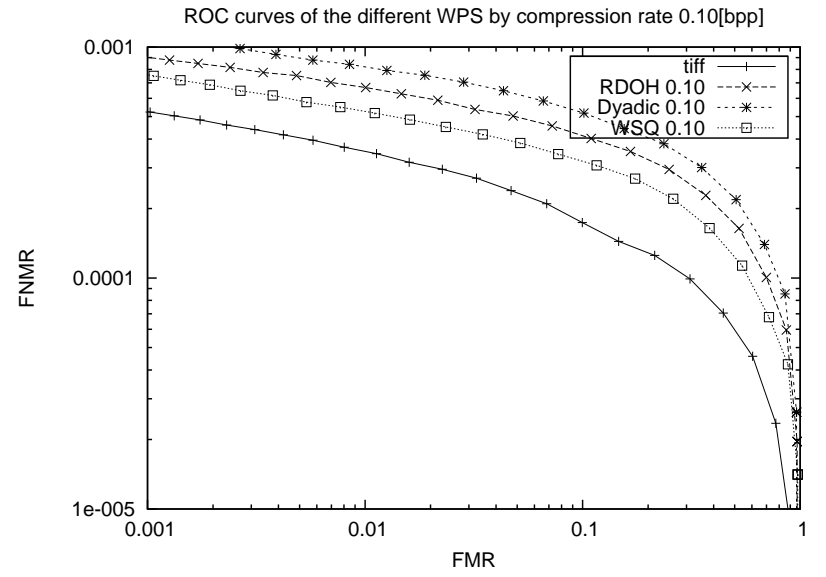

(b) DB2

Figure 6. CCC scenario: ROC of the RDOH, Dyadic and WSQ WP subband structures at rate 0.1 [bpp].

We now turn to the results for the CUC scenario (i.e. matching a compressed sample against uncompressed 
database entries). In Fig. 7 results for DB1 and DB2 at bitrate 0.1 [bpp] are shown.

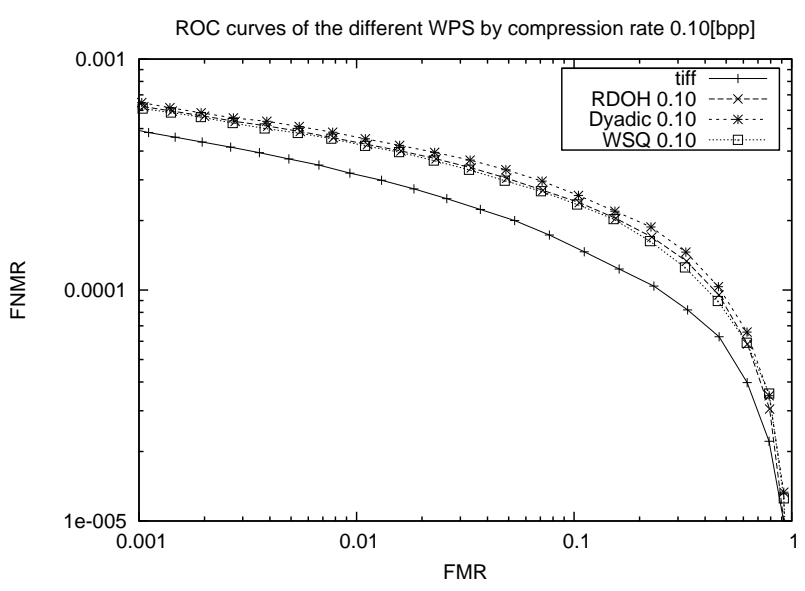

(a) DB1

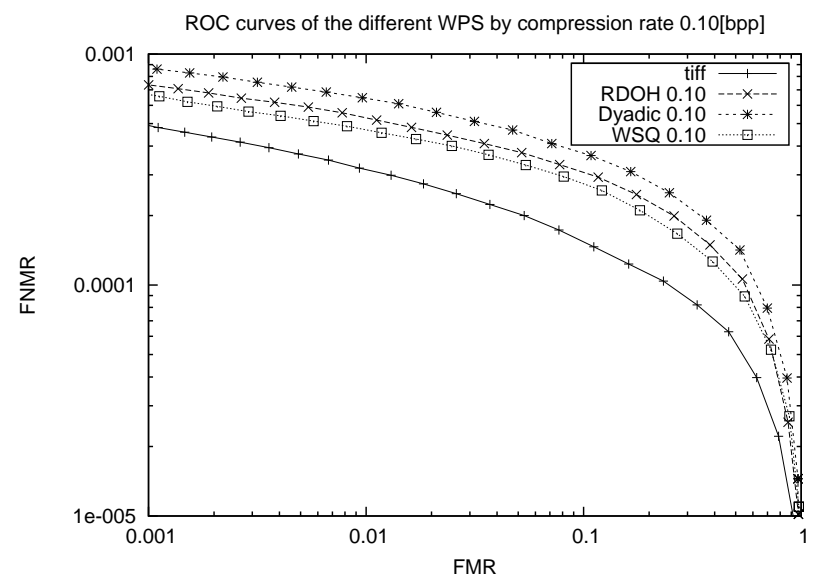

(b) DB2

Figure 7. CUC scenario: ROC behaviour of the RDOH, Dyadic and WSQ WP subband structures at bitrate 0.1 [bpp]

For DB1, results are somewhat close together but the Dyadic structure is always worst. While RDOH and WSQ perform almost identical, WSQ is slightly superior for $F M R>0.1$. The situation is as clear as in the CCC case for DB2 (with WSQ performing best and Dyadic worst), but the RDOH behaviour is closer to WSQ here.

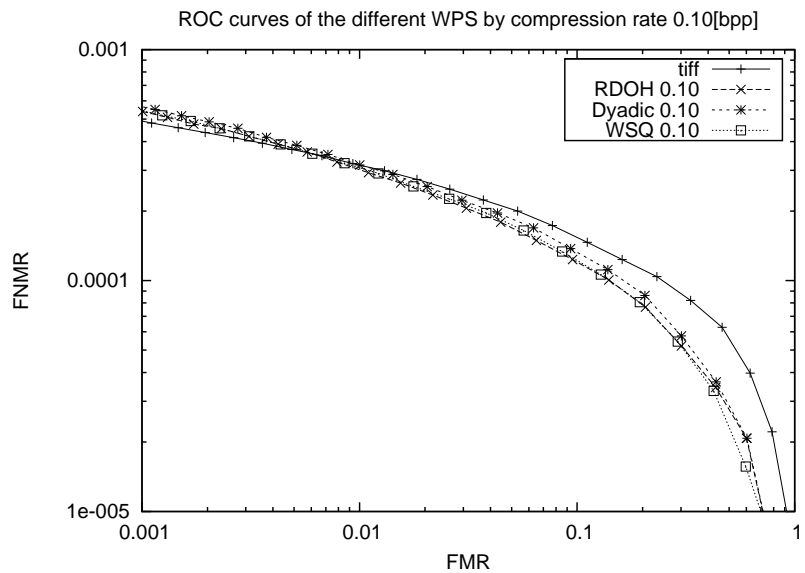

(a) DB3

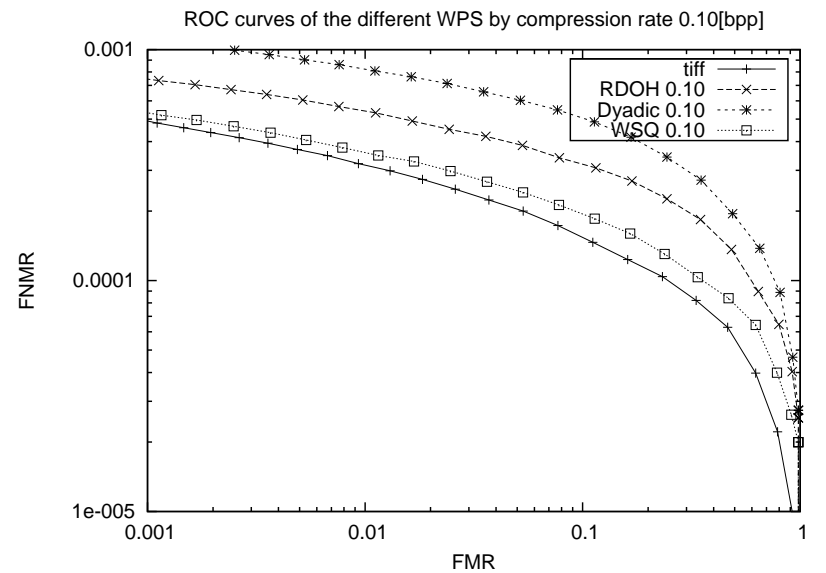

(b) DB4

Figure 8. CUC scenario: ROC behaviour of the RDOH, Dyadic and WSQ WP subband structures at bitrate 0.1 [bpp]

The matching result for DB4 as shown in Fig. 8.b is similar to that of DB2, but even more pronounced. For DB3 (Fig. 8.a) we observe the interesting effect that for $F M R>0.01$, all compression schemes deliver better matching performance as compared to the uncompressed case. This phenomenon has been observed before for some biometric data types and is due to the fact that compression algorithms act as some sort of low-pass filter resulting in a limited denoising capability (which is able to improve the results for some types of data). However, this is somewhat unexpected at a low bitrate like the one considered here. Also in this case, WSQ and RDOH outperform the Dyadic WP structure slightly. 


\subsubsection{Interpretation}

We have found significant differences among the three compression techniques considered for lower bitrates and a subset of the databases considered only. For medium and higher bitrates, the performance is almost identical. Consequently, the use of wavelet packet subband structures in JPEG2000 only results in recognition advantages in case of severe compression being applied.

For the lower bitrates mentioned (i.e. $0.1-0.2$ [bpp]), we have discovered a discrepancy between the image "quality" measure PSNR and the actual recognition performance in case the compressed images are used in the matching process. While the rate-distortion performance of the RDOH WP subband structure selection scheme always produces the best PSNR values (which is obvious due to construction), matching performance is often better for the WSQ structure, which on the other hand exhibits the lowest PSNR performance of all three schemes. The dyadic structure, often being quite close to the leading RDOH scheme in terms of rate-distortion performance, exhibits the worst recognition results. Overall, PSNR is found to be a hardly reliable indicator for matching performance of the fingerprint recognition system considered in case of low bitrates.

We have found the two considered scenarios, CUC and CCC, to result in almost identical trends with respect to the ranking of the compression schemes under investigation. However, in terms of absolute values, CCC results are slightly better. Therefore, it can be recommended to compress both images involved in matching in case compression has to be applied. For one database (DB3), we have even been able to improve on the results of uncompressed imagery.

\section{CONCLUSIONS AND FUTURE WORK}

Significant differences among different wavelet-packet based compression schemes in JPEG2000 have been observed for lower bitrates only. In these cases, we have found PSNR to be a poor indicator of finger recognition matching scores. In particular, adaptive, rate-distortion optimised subband structures do not lead to improved recognition behaviour while the fixed WSQ scheme turns out to deliver excellent matching performance also in the JPEG2000 environment.

In future we will cover two issues. First, more and different fingerprint recognition systems will be considered. So far, we do not know if the results do carry over to different types of fingerprint recognition schemes, e.g. not based on minutiae matching. Second, we will develop an algorithm for WP basis choice optimising matching performance instead of rate-distortion behaviour.

\section{Acknowledgements}

The work described in this paper is partially supported by the Austrian Science Fund, TRP project no. L554.

\section{REFERENCES}

1. W. Fong, S. Chan, and K. Ho, "Designing JPEG quantization matrix using rate-distortion approach and human visual system model," in Proceedings of the IEEE International Conference on Communications (ICC'97), 3, pp. 1659-1663, 1997.

2. M. Chen, S. Zhang, and M. Karim, "Modification of standard image compression methods for correlationbased pattern recognition," Optical Engineering 43(8), pp. 1723-1730, 2004.

3. G.-M. Jeong, C. Kim, H.-S. Ahn, and B.-J. Ahn, "JPEG quantization table design for face images and its application to face recognition," IEICE Transactions on Fundamentals of Electronics, Communications and Computer Science E69-A(11), pp. 2990 - 2993, 2006.

4. M. Konrad, H. Stögner, and A. Uhl, "Custom design of JPEG quantization tables for compressing iris polar images to improve recognition accuracy," in Proceedings of the 3rd International Conference on Biometrics 2009 (ICB'09), M. Tistarelli and M. Nixon, eds., LNCS 5558, pp. 1091-1101, Springer Verlag, 2009.

5. M. Konrad, H. Stögner, and A. Uhl, "Evolutionary optimization of JPEG quantization tables for compressing iris polar images in iris recognition systems," in Proceedings of the 6th International Symposium on Image and Signal Processing and Analysis, ISPA '09, (Salzburg, Austria), Sept. 2009. 
6. G. Kostmajer, H. Stögner, and A. Uhl, "Custom JPEG quantization for improved iris recognition accuracy," in Emerging Challenges for Security, Privacy and Trust. Proceedings of the 24th IFIP International Information Security Conference 2009 (IFIP SEC'09), D. Gritzalis and J. Lopez, eds., IFIP AICT 297, pp. 76-86, Springer Verlag, May 2009.

7. J. N. Bradley, C. M. Brislawn, and T. Hopper, "The FBI wavelet/scalar quantization standard for gray-scale fingerprint image compression," in SPIE Proceedings, Visual Information Processing II, 1961, pp. 293-304, (Orlando, FL, USA), Apr. 1993.

8. M. Wickerhauser, Adapted wavelet analysis from theory to software, A.K. Peters, Wellesley, Mass., 1994.

9. D. Taubman and M. Marcellin, JPEG2000 - Image Compression Fundamentals, Standards and Practice, Kluwer Academic Publishers, 2002.

10. R. Coifman and M. Wickerhauser, "Entropy based methods for best basis selection," IEEE Transactions on Information Theory $\mathbf{3 8}(2)$, pp. 719-746, 1992.

11. C. Taswell, "Satisficing search algorithms for selecting near-best bases in adaptive tree-structured wavelet transforms," IEEE Transactions on Signal Processing 44, pp. 2423-2438, Oct. 1996.

12. T. Schell and A. Uhl, "Optimization and assessment of wavelet packet decompositions with evolutionary computation," EURASIP Journal on Applied Signal Processing 2003(8), pp. 806-813, 2003.

13. K. Ramchandran and M. Vetterli, "Best wavelet packet bases in a rate-distortion sense," IEEE Trans. on Image Process. 2(2), pp. 160-175, 1993.

14. N. M. Rajpoot, R. G. Wilson, F. G. Meyer, and R. R. Coifman, "Adaptive wavelet packet basis selection for zerotree image coding," IEEE Transactions on Image Processing 12(12), pp. 1460-1472, 2003.

15. Y. Yang and C. Xu, "A wavelet packet based block-partitioning image coding algorithm with rate-distortion optimization," in Proceedings of the IEEE International Conference on Image Processing (ICIP'05), III, pp. 201-204, IEEE, 2005.

16. Y. Yang and C. Xu, "Fast and efficient basis selection methods for embedded wavelet packet image coding," in Proceedings of Third International Conference of Image Analysis and Recognition, ICIAR'06, Lecture Notes in Computer Science 4141, pp. 480-492, Springer-Verlag, Sept. 2006.

17. M. Reisecker and A. Uhl, "Wavelet-packet subband structures in the evolution of the JPEG2000 standard," in CD-ROM Proceedings of the 6th IEEE Nordic Signal Processing Symposium (NORSIG 2004), IEEE Norway Section, (Espoo, Finland), June 2004.

18. D. Engel and A. Uhl, "Secret wavelet packet decompositions for JPEG2000 lightweight encryption," in Proceedings of 31st International Conference on Acoustics, Speech, and Signal Processing, ICASSP '06, V, pp. 465-468, IEEE, (Toulouse, France), May 2006.

19. W. Pennebaker and J. Mitchell, JPEG - Still image compression standard, Van Nostrand Reinhold, New York, 1993.

20. M. A. Lepley, "Profile for 1000ppi fingerprint compression," Tech. Rep. MTR 04B0000022, The MITRE Corporation, 2004.

21. R. Kidd, "Comparison of wavelet scalar quantization and JPEG for fingerprint image compression," Journal of Electronic Imaging 4(1), pp. 31-39, 1995.

22. B. G. Sherlock and D. M. Monro, "Optimized wavelets for fingerprint compression," in Proceedings of the 1996 International Conference on Acoustics, Speech and Signal Processing (ICASSP'96), (Atlanta, GA, USA), May 1996.

23. B. G. Sherlock and D. M. Monro, "Psychovisually tuned wavelet fingerprint compression," in Proceedings of the IEEE International Conference on Image Processing (ICIP'96), IEEE Signal Processing Society, (Lausanne, Switzerland), Sept. 1996.

24. T.-K. Lin, "Wavelet compression of fingerprints and recognition using moment invariants," in IASTED Conference on Artificial Intelligence and Applications, (Innbruck, Austria), Feb. 2006. paper no. 502-031.

25. O. Gerek and A. Cetin, "Polyphase adaptive filter banks for fingerprint image compression," Electronics Letters 34, pp. 1931-1932, Oct. 1998.

26. B. G. Sherlock and D. M. Monro, "Balanced uncertainty wavelets for fingerprint compression," in IEE Colloquium on Image Processing for Security Applications, pp. 5-8, (London, GB), Mar. 1997. 
27. U. Grasemann and R. Miikkulainen, "Effective image compression using evolved wavelets," in GECCO '05: Proceedings of the 2005 conference on Genetic and evolutionary computation, pp. 1961-1968, ACM Press, (New York, NY, USA), 2005.

28. J. Scharinger, H. Gaderbauer, and G. Wilflingseder, "Redesigning the FBI fingerprint compression standard," in Pattern Recognition and Medical Computer Vision 1998, M. Gengler, M. Prinz, and E. Schuster, eds., books@ocg.at 106, pp. 215-223, Austrian Computer Society, 1998.

29. S. Kasaei, M. Deriche, and B. Boashash, "A novel fingerprint image compression technique using wavelet packets and pyramid lattice vector quantization," IEEE Transactions on Image Processing 12(11), pp. 13651378, 2002.

30. P. Saeedian and B. Shirazi, "A novel fingerprint image compression technique using adaptive subband image coding," in Proceedings of the 2004 Picture Coding Symposium PCS'04, 2004.

31. G. Khuwaja, "Best parameter based compression of fingerprints with wavelet packets," International Journal of Computer Applications in Technology 19(1), pp. 51-62, 2004.

32. J. Thärna, K. Nilsson, and J. Bigun, "Orientation scanning to improve lossless compression of fingerprint images," in Proceedings of AVBPA, J. Kittler and M. Nixon, eds., LNCS 2688, pp. 343-350, Springer Verlag, 2003.

33. R. Sudhakar, N. Vigesh, and S. Jayaraman, "Application of wavelet footprints for fingerprint compression," ICGST International Journal on Graphics, Vision, and Image Processing 5(6), pp. 39-45, 2005.

34. A. Said and W. A. Pearlman, "A new, fast, and efficient image codec based on set partitioning in hierarchical trees," IEEE Transactions on Circuits and Systems for Video Technology 6, pp. 243-249, June 1996.

35. R. Sudhakar, R. Karthiga, and S. Jayaraman, "Fingerprint compression using contourlet transform with modified SPIHT algorithm," IJECE Iranian Journal of Electrical and Computer Engineering 5(1), pp. 3-10, 2005.

36. R. Eslami and H. Radha, "Wavelet-based contourlet transform and its application to image coding," in Proceedings of the IEEE International Conference on Image Processing (ICIP'04), IEEE Signal Processing Society, (Singapore), Oct. 2004.

37. R. Eslami and H. Radha, "Wavelet-based contourlet packet image coding," in 2005 Conference on Information Sciences and Systems, (The John Hopkins University), Mar. 2005.

38. C. Beleznai, H. Ramoser, B. Wachmann, J. Birchbauer, H. Bischof, and W. Kropatsch, "Memory-efficient fingerprint verification," in Proceedings of the IEEE International Conference on Image Processing (ICIP'01), 2, pp. 463-466, (Thessaloniki, Greece), Oct. 2001.

39. A. Mascher-Kampfer, H. Stögner, and A. Uhl, "Comparison of compression algorithms' impact on fingerprint and face recognition accuracy," in Visual Communications and Image Processing 2007 (VCIP'07), C. Chen, D. Schonfeld, and J. Luo, eds., Proceedings of SPIE, pp. 650810-1 - 65050N-10, SPIE, (San Jose, CA, USA), Jan. 2007.

40. W. Funk, M. Arnold, C. Busch, and A. Munde, "Evaluation of image compression algorithms for fingerprint and face recognition systems," in Proceedings from the Sixth Annual IEEE Systems, Man and Cybernetics (SMC) Information Assurance Workshop, J. Cole and S. Wolthusen, eds., pp. 72-78, IEEE Computer Society, June 2006.

41. C. I. Watson, M. D. Garris, E. Tabassi, C. L. Wilson, R. M. McCabe, and S. Janet, "User's guide to nist fingerprint image software 2 (nfis2)." http://fingerprint.nist.gov/NFIS/. 